\title{
Quelles logiques d'innovation des enseignants universitaires développent-ils en réponse à un appel institutionnel à projets ?
}

\author{
JOHAN TIRTIAUX \\ Université de Namur, Service de Pédagogie Universitaire, \\ johan.tirtiaux@unamur.be \\ MARC ROMAINVILLE \\ Université de Namur, Service de Pédagogie Universitaire \\ marc.romainville@unamur.be
}

\section{Résumé}

Par l'analyse de projets soumis en réponse à un appel institutionnel à expériences innovantes, cet texte interroge les motivations qui poussent les enseignants à l'innovation à l'université et les formes que prennent les dispositifs pédagogiques proposés. Nous dégageons quelques logiques d'innovation et tentons d'en saisir l'importance parmi les projets soumis ainsi que leur articulation.

\begin{abstract}
Through the analysis of projects submitted in response to an institutional call for innovative experiences, this paper questions the motivations that drive university teachers to innovation and the forms that the proposed pedagogical devices take. We identify some logics of innovation and attempt to understand their importance among the projects submitted as well as their articulation.
\end{abstract}

\section{Mots-clés}

Innovation, méthodes pédagogiques, compétences, institutions et politiques éducatives 


\section{Key words}

Innovation, teaching methods, skills, educational institutions and policies

\section{Introduction}

On constate aujourd'hui que les pratiques enseignantes universitaires sont en mutation (RegeColet et Romainville, 2006). Cette dynamique se traduit notamment par un intérêt marqué aujourd'hui pour l'innovation pédagogique. Rege-Colet (2007) a identifié quatre tendances de l'innovation pédagogique à l'université : 1. La pédagogie par objectifs et la construction d'unités d'enseignement autour des objectifs à atteindre ; 2. les méthodes d'enseignements avec l'introduction de l'enseignement par projet, l'apprentissage par problèmes et de façon générale l'introduction de méthodes interactives qui sollicitent une participation accrue des étudiants ; 3. des modalités d'évaluation des acquis faisant appel à des situations authentiques ou à l'usage du portfolio ; 4. l'intégration des technologies dans l'enseignement et le recours à l'e-learning. Roegiers (2012) ajoute la tendance au rapprochement de l'université à la sphère professionnelle. Lison et al. (2014), sur la base d'une analyse de 11 programmes au Canada, dégagent quant à eux six caractéristiques «d'un dispositif curriculaire innovant»: (1) centré sur l'étudiant, proposant (2) une contextualisation des apprentissages, (3) une réduction du cloisonnement disciplinaire, (4) une évaluation cohérente avec l'esprit de l'innovation, il met (5) l'accent curriculaire sur le transfert des apprentissages et favorise (6) la collégialité entre les professeurs. On le voit, la mutation de l'enseignement universitaire suit des tendances hétérogènes qu'on ne saurait résumer à la dimension technologique. Si Béchard et Pelletier (2001, p. 133) définissent l'innovation pédagogique dans l'enseignement supérieur comme une «action délibérée qui cherche à introduire de la nouveauté dans un contexte donné dans le but d'améliorer substantiellement les apprentissages des étudiants, en situation d'interaction et d'interactivité », Cros (2004) identifie quelques caractéristiques essentielles de l'innovation pédagogique : la perspective de la nouveauté, le phénomène de changement conscient et volontaire, l'action finalisée vers certains buts et un processus inscrit dans une temporalité. Elle précise que «l'innovation résulte d'une intention et met en ouvre une ou des actions visant à changer ou modifier quelque chose (un état, une situation, une pratique, des méthodes, un fonctionnement), à partir d'un diagnostic d'insuffisance, d'inadaptation ou d'insatisfaction par rapport aux objectifs à atteindre, aux résultats, aux relations de travail »(Cros, 2001). Ce diagnostic peut porter sur les apprentissages des étudiants, mais pas seulement. Est-ce 
uniquement l'amélioration de l'apprentissage qui sous-tend les velléités de changement ? Quelles insuffisances de l'enseignement universitaire poussent les enseignants à innover ? Et quelles formes prennent les innovations en regard de ces insuffisances ?

Le présent article vise à identifier les motivations et les formes que prennent des projets d'innovations pédagogiques qui répondent à un appel à projet innovant émis par une université. En adoptant le point de vue des acteurs enseignants, nous relèverons les raisons qui les incitent à innover en analysant les insuffisances ou lacunes perçues dans l'enseignement universitaire, et en regard de ces raisons, nous tenterons de dégager les formes que doivent prendre les dispositifs innovants pour répondre à ces difficultés. L'objectif est de saisir, au travers de ces projets soumis en réponse à l'appel institutionnel, quelques logiques qui travaillent les professeurs qui aspirent à innover.

Après avoir décrit l'appel à projet source du matériau empirique exploité ici, cet article présentera les questions de recherche ainsi que la méthodologie adoptée. Il abordera ensuite les résultats de ces analyses, à savoir les cinq logiques d'innovations pédagogiques dégagées et leur importance au sein des projets analysés. Le texte se termine sur une discussion de ces résultats pointant certaines questions plus spécifiques (limites majeures de l'enseignement universitaire identifiées, présence du numérique...).

\section{L'appel à projet $\mathrm{PUNCH}$}

Le projet PUNCH Pédagogie Universitaire Namuroise en CHangement réside en un appel à projets innovants. Des financements ont été dégagés afin de soutenir des expériences pilotes durant trois ans. Au terme de cette période et d'une évaluation des projets, des conclusions seront tirées et une étape d'extension et de généralisation, appuyée sur les dynamiques innovationnelles les plus prometteuses, est prévue. Ces projets pilotes sont donc soutenus dans la perspective de redéfinir la pédagogie de demain de notre institution. Cette visée de généralisation distingue le projet PUNCH des habituels fonds de soutien à l'innovation. Il est intéressant de préciser rapidement l'origine de ce projet. Roegiers (2012, p. 102) souligne que, parmi les facteurs qui poussent à des réformes curriculaires, des préoccupations de nature identitaire jouent souvent un rôle important : «Les transformations des curricula sont (...) vues comme une opportunité de clarifier le projet de l'institution : l'amener à améliorer son image pour mieux la positionner dans l'éventail des offres de formation ». Ce facteur survient parfois à la suite d'une fusion d'institutions ou au contraire d'une séparation. Le projet PUNCH donne raison à cette thèse. Cette dynamique de réforme est en effet née de l'échec d'un projet de 
fusion, notamment initiée par une politique de regroupement des institutions d'enseignement supérieur en Belgique francophone. Cette fusion avortée a fait naître un questionnement identitaire de notre institution sur elle-même : quel est notre projet spécifique d'enseignement? Un vaste chantier visant à « définir un projet d'Université » et à « renforcer notre identité et affirmer nos différences » [extraits de documents internes] a été mis en œuvre. Des groupes de réflexions ont été créés autour de thèmes spécifiques : qualité, recherche etc. Parmi ces chantiers, le projet PUNCH est né pour répondre à ces questions : Quelles sont les lignes directrices de notre pédagogie ? Quel sera notre enseignement de demain ? Précisons que l'appel à projet suggérait six dimensions générales d'innovation : 1. oser être innovant ; 2. définir davantage collectivement les finalités d'apprentissage ; 3. favoriser l'intégration des enseignements et rompre avec le cloisonnement disciplinaire ; 4. recourir de façon volontariste aux méthodes actives ; 5. développer une évaluation intégrée, continue et formative ; 6 . veiller au caractère transférable du projet.

\section{Questions de recherche et méthodologie}

L'analyse vise à dégager les grandes logiques qui caractérisent ces projets d'innovation et à mesurer l'ampleur de chacune d'entre elles. Par «logique d'innovation », nous entendons cerner de façon idéal-typique quelques grandes tendances des projets proposés au départ de deux questions : 1. pourquoi les enseignants jugent-ils opportun de proposer un projet d'innovation pédagogique (difficultés et insuffisance ressenties, changements espérés...) ? 2. Comment projettent-ils d'innover (caractéristiques des dispositifs, niveau d'intervention)?

Sur le plan méthodologique, l'analyse s'appuie sur trois matériaux : les 20 dossiers soumis, 10 entretiens (enregistrés) réalisés avec des promoteurs de projets et des échanges informels avec ces promoteurs dont le contenu a été consigné. Les échanges informels et les entretiens portaient sur les insuffisances perçues de l'enseignement universitaire, sur les motivations à changer et sur les solutions, qu'il s'agisse de dispositifs pédagogiques, curriculaires ou règlementaires. La technique d'analyse de contenu s'appuie sur l'approche par catégories conceptualisantes de Paillé et Mucchielli (2008) croisée à l'approche typologique de Schnapper (1999). Quatre grandes étapes caractérisent cette analyse :

1. Un premier découpage du matériau sur la base de deux critères : les motivations exprimées qui ont incité à proposer le projet (difficultés identifiées et buts poursuivis) et les caractéristiques du dispositif. 
2. Les extraits identifiés à l'étape $n^{\circ} 1$ ont ensuite été analysés via un premier codage visant à synthétiser par différents items (souvent repris des termes des promoteurs eux-mêmes) les multiples unités de sens qui caractérisent les motivations (Ex.: «diminuer le cloisonnement disciplinaire », «favoriser des savoirs mobilisés», «lutter contre l'échec» ...) et les dispositifs proposés («intégrer plusieurs enseignements», «travailler sur la base de cas réels » ...).

3. Un travail de rapprochement et de classement des items dégagés dans la deuxième étape autour de catégories conceptualisantes (des idéaux-types, dirait Schnapper) qui «décollent » des mots des acteurs et prétendent saisir une cohérence derrière une diversité d'items et se distinguer d'autres principes fondamentaux. Ici, cinq logiques d'innovation ont été dégagées.

4. Un travail de confrontation systématique de l'ensemble du matériau aux logiques dégagées, permettant à la fois de quantifier la présence de ces logiques dans les projets et de montrer que les projets relèvent parfois de plusieurs logiques.

\section{Résultats : cinq logiques d'innovation pédagogique}

Au terme de l'analyse, il apparaît que cinq logiques sous-tendent les projets :

1. logique de libération de l'innovation;

2. logique de remédiation;

3. logique des compétences ;

4. logique de l'intégration (des enseignements et des apprentissages) ;

5. logique de la citoyenneté.

Une première distinction doit d'emblée être établie entre les logiques ayant trait aux processus de développement de l'innovation pédagogique, c'est-à-dire de ce qui la freine ou la favorise, et celles qui concernent les contenus des dispositifs curriculaires ou pédagogiques. La première logique présentée ci-dessous se rapporte à la dimension «processus », là où les suivantes se situent du côté des contenus.

\subsection{La logique de libération de l'innovation}

Une première logique, qui touche deux projets, consiste à favoriser le changement dans les méthodes pédagogiques universitaires et dans les façons d'apprendre des étudiants. La difficulté ressentie tient à l'idée que l'enseignement innove insuffisamment, est peu créatif et reste trop cantonné à des schémas éculés dont le cours magistral apparaît comme l'exemple 
type. «Il faut arrêter d'enseigner comme au Moyen Âge », souligne un enseignant pour qui «le cours magistral ne peut plus être qu'une activité d'apprentissage parmi d'autres ». Un autre projet veut favoriser «l'expérimentation de voies nouvelles en matière de création, d'appropriation et de transfert des savoirs que nous enseignons ». Nous sommes proches de la définition de l'innovation pédagogique de Bédard et Béchard, qui considèrent comme innovant « tout ce qui ne relève pas de l'enseignement magistral, méthode encore utilisée par une très grande majorité de professeurs » (2009, p. 258). Parmi les arguments justifiant l'éloignement du cours magistral, les dossiers mentionnent l'accès universel au savoir par internet, la concurrence interuniversitaire européenne, le regret de la «position de retrait de soi trop souvent adoptée par les étudiants».

Nous l'avons dit, cette logique consiste non pas à proposer une innovation pédagogique ou curriculaire, mais à intervenir sur les conditions d'émergence de l'innovation. Elle ne propose aucune indication sur la forme précise que devrait prendre l'enseignement de demain. Deux projets sont ici concernés. Lesquels proposent des moyens différenciés de favoriser la libération de l'innovation. L'un joue sur les règles institutionnelles, l'autre sur la culture pédagogique.

Le premier projet part de l'idée que ce qui freine l'innovation c'est le lien contractuel qui lie un enseignant à l'obligation de prester un certain nombre d'heures de cours : «Dans notre université [...], la seule unité servant à comptabiliser la charge d'encadrement d'un académique est l'heure de cours. Ce mode de comptabilisation encourage à recourir de façon massive à l'enseignement ex cathedra et rend très difficile, sinon impossible [...] d'imaginer, élaborer et mettre en œuvre des processus d'apprentissages novateurs. ». Le projet suggère de passer d'une comptabilisation en heures de cours à une comptabilisation en heures d'aide à l'apprentissage qui autorisera d'autres activités pédagogiques que le cours magistral.

Le second projet se propose de faire évoluer la culture pédagogique en proposant des formations à la créativité. Des séminaires animés par des spécialistes de la créativité doivent favoriser l'émergence d'autres façons plus créatives d'enseigner et d'apprendre : «Partant des cadres conceptuels que nous enseignons, le projet propose des outils, des méthodes et des échanges pour façonner des projets créatifs qui font parler ces cadres, les interrogent, les dépassent, les travaillent comme un auteur de roman peut le faire avec des mots et une grammaire. »Ces séminaires feront émerger «de nouveaux projets et dégager les formes adéquates pour ouvrir davantage nos étudiants à une pensée qui intègre la créativité et l'inventivité ». 


\subsection{La logique de l'E-remédiation}

Une deuxième logique s'appuie sur le principe de l'E-remédiation. Cinq projets sont concernés. À la différence des autres logiques, les points de départ sont ici les échecs et difficultés d'apprentissage des étudiants. Un professeur regrette la difficulté à acquérir la méthode du raisonnement mathématique. Un autre souligne la difficulté à manipuler les microscopes et à interpréter les observations. Un autre encore évoque la non-maîtrise des notions de base de méiose ou de mitose. Tous les projets concernés portent sur des cours de première année. Ce n'est pas un hasard en Belgique francophone où les taux d'échec y sont importants (plus de 60 $\%)$.

La logique de l'E-remédiation vise à pallier ces difficultés en proposant aux étudiants des ressources et des dispositifs nouveaux. Ces derniers s'appuient tantôt exclusivement, tantôt partiellement, sur des outils en ligne d'auto-apprentissage. La dimension technologique est donc importante ; c'est la raison pour laquelle nous avons parlé d'«E-remédiation » et non simplement de remédiation. Ces dispositifs ne remettent par conséquent nullement en cause le fonctionnement des cours eux-mêmes. Parfois, ils sont articulés à des séances en présentiel portées tantôt par une cellule d'appui pédagogique extérieure aux cours tantôt par les travaux dirigés associés aux cours.

Les dispositifs d'auto-apprentissage proposés ici sont de nature variable : podcast de cours (Powerpoint commentés) destinés à transmettre une aide sur la méthode de raisonnement de la discipline, QCM en ligne, plateforme d'aide à la remédiation des lacunes langagières et logiciel d'utilisation d'un microscope.

\subsection{La logique des compétences}

Une autre logique caractérise des projets qui s'appuient sur une critique du caractère superficiel des apprentissages des étudiants. Un professeur constate un taux d'échec accru après être passé de son traditionnel questionnaire à choix multiples (QCM) à un examen à questions ouvertes. Un autre enseignant regrette que : «les savoirs acquis restent théoriques voire livresques ». Cette maîtrise «superficielle » des apprentissages est associée aux méthodes magistrales dont ces projets vont s'éloigner.

Nous avons qualifié cette famille de motivations (et de dispositifs liés) de «logique des compétences ». La notion de compétence prend certes des significations variables dans les projets pédagogiques (Roegiers, 2012). Certains (Romainville, 2008 ; Tardif, 2006) considèrent 
toutefois qu'elle renvoie en pédagogie à une idée centrale, celle d'un « savoir vivant », c'est-àdire d'un ensemble de « ressources diverses (savoirs, savoir-faire et attitudes) que la personne « compétente » est capable de mobiliser pour affronter avec efficacité une situation ou résoudre un problème » (Romainville, 2008, p. 36). La logique des compétences appelle des méthodes actives, c'est-à-dire appuyées précisément sur des situations dans lesquelles les étudiants ont à mobiliser ces ressources. Parmi ces projets, les expressions «compétences»», «mobilisation de savoirs», «manipulation des concepts», «appropriation active des savoirs », reviennent régulièrement.

Dix projets s'inscrivent dans cette logique. Ils proposent des dispositifs favorisant la mise en pratique des notions par confrontation à des «situations complexes ». Ils considèrent que les dispositifs actifs favoriseront un «apprentissage en profondeur», une «consolidation des connaissances », une «meilleure rétention » et également une meilleure «motivation» des étudiants. Certains visent également des compétences «nouvelles» et «transversales»: "apprendre à se poser des questions », «innover», "problématiser», "travailler en groupe », « acquérir des compétences réflexives ».

La logique des compétences s'arrime également à une volonté de « rapprocher la formation universitaire du monde professionnel » et ce, sous des formes variables selon les projets : cas authentiques ou semi-authentiques, stage, jury avec professionnels invités, consultation de professionnels dans la construction de référentiels de compétence.

L'innovation réside donc dans des dispositifs pédagogiques destinés à se substituer aux méthodes d'enseignement classiques. Les méthodes dans les projets proposées sont variables : approche par projet en physique $(n=1)$, team based learning en anatomie animale $(n=1)$, classe inversée en médecine ou en langue $(n=3)$, analyse de cas en pharmacie, en histoire, en didactique, en psychologie (souvent sur base de documents vidéos) $(n=4)$, construction d'une problématique sur base d'une expérience pratique de stage en communication $(n=1)$. De façon générale, les projets appartenant à cette logique se situent plutôt au niveau de la deuxième ou de la troisième année $(n=7)$, voire au-delà $(n=1)$. Quelques projets $(n=3)$ relèvent le défi de développer cette pédagogie active en première année.

\subsection{La logique de l'intégration}

Intégrer signifie « assembler des parties pour former un tout». Ce principe visant à atteindre une cohérence globale au départ d'une diversité d'éléments est le fondement de la logique d'intégration portée par six projets. La difficulté ressentie ici tient à un sentiment de manque 
de liens et de cohérence entre les cours et d'un cloisonnement excessif des enseignements. Un enseignant souligne que le programme dans lequel il enseigne est avant tout une "série de cours qui [...] fonctionnent en vase clos et donnent lieu à des évaluations distinctes ». Il regrette le «trop peu de connexions [...] établies entre les enseignements par les professeurs». Ce cloisonnement est généralement associé à un cloisonnement des apprentissages chez les étudiants et à une capacité insuffisante à mobiliser des acquis d'un cours (souvent de cours de base du premier baccalauréat) dans d'autres cours. «En général, les étudiants cloisonnent les différents enseignements lors du processus d'apprentissage. L'objectif de ce projet est de lutter contre cette tendance. »Un autre avance que «les étudiants apprennent par tiroir ». Ils sont «peu capables d'établir le lien entre un cours de Bac2 et Bac3 et les cours de base ». Ce constat est posé dans des disciplines aussi diverses que les sciences vétérinaires, les sciences pharmaceutiques, l'histoire ou la communication.

Le cœur du principe des dispositifs proposés est l' «intégration des enseignements » avec en point de mire l'idée d' «aider les étudiants à intégrer les différents apprentissages ». Il s'agit, comme le mentionne un projet, de «renforcer les ponts entre différents cours », de «mieux intégrer les enseignements dans un projet de formation», de favoriser le «décloisonnement des cours ».

En réalité, ce décloisonnement s'appuie sur des dispositifs variés. Une première façon d'intégrer consiste à créer un cours transversal. Ainsi, un enseignant propose de consulter ses collègues de première et troisième année pour identifier les notions abordées et jugées nécessaires (PH, lipophilie, solubilité, rigueur scientifique...) pour les incorporer à son cours d'analyse pharmaceutique. Il crée ce qu'il nomme un «cours transversal » qui vise à «faire le pont entre sciences de base (chimie, physique, biologie), sciences médicales et pharmaceutiques ».

Une deuxième façon d'intégrer les enseignements consiste à redéfinir collectivement le programme de cours en précisant les finalités communes poursuivies. Ainsi, un projet se propose de «faire évoluer un ensemble atomisé de cours confiés à des professeurs différents vers un enseignement global de compétences transversales encadré par une équipe pédagogique ». La logique de l'intégration s'approche ici de l'approche-programme (Prégent et al., 2009). Concrètement, cela passe par la définition d'un socle commun de compétences visées, des harmonisations notamment sur le plan conceptuel et un meilleur agencement des cours sous forme de modules cohérents. 
Une troisième façon d'intégrer les enseignements revient à favoriser des analyses et des travaux interdisciplinaires réalisés à la jonction de plusieurs cours. Des professeurs de disciplines différentes travaillent de concert sur un même objet. Par exemple, des enseignants de didactique générale travaillent à l'analyse de cas réels (sous forme de vidéos) en même temps que les didacticiens disciplinaires. Autre exemple, des professeurs de langage du cinéma, d'histoire du cinéma et de sociologie réalisent des séances d'analyses croisées autour d'un même document audiovisuel. Certains de ces projets proposent des évaluations intégrées où la maîtrise de plusieurs disciplines est évaluée lors d'une épreuve unique. D'autres travaillent un même support dans des temporalités distinctes : une première analyse d'un objet est réalisée dans un cours qui sera ensuite repris dans un autre cours.

Une quatrième façon de promouvoir l'intégration est la création de parcours pédagogiques interdisciplinaires en ligne. Un projet propose la mise sur pied d'une plateforme web établissant une jonction entre sciences de base et physiologie animale. Les parcours pédagogiques proposés possèdent deux entrées et serviront à illustrer l'intérêt des sciences de base en première année par des vidéos de cas d'animaux et à permettre le rappel des notions de base en physiologie animale dans les années qui suivent.

Enfin, cinquième méthode proposée, l'intégration des enseignements aboutit, dans certains projets, à un travail intégrateur de fin de cycle en troisième année (fin du premier cycle de baccalauréat). Les étudiants doivent montrer qu'ils y mobilisent les compétences acquises dans les différents cours du cursus et le jury est pluridisciplinaire. Il s'agit, par exemple, de l'analyse d'une expérience de stage appuyée sur des notions acquises au fil de la formation et présentée en trois langues devant un jury composé des enseignants de la filière et de professionnels.

\subsection{La logique de la citoyenneté}

Une dernière logique se distingue par une volonté de soutenir, parallèlement aux missions d'apprentissages disciplinaires et de diplomation poursuivies par les universités, une formation à une citoyenneté critique associée à la transmission de valeurs humanistes. Un seul projet relève de cette logique spécifique. «Le projet a pour ambition [...] de rappeler que l'enseignement universitaire [...] ne concerne pas seulement une somme de connaissances et l'acquisition de savoir-faire, mais également une réflexion et une prise de distance critique au sujet des enjeux fondamentaux de la condition humaine et des grands problèmes sociétaux [...] en particulier celui de l'inégalité entre les hommes et entre les peuples. » L'insuffisance pointée ici concerne une focalisation excessive de l'enseignement universitaire sur une dimension 
instrumentale (transmission de savoirs, de compétences et de techniques). Une place jugée insuffisante est faite aux activités génératrices d'interrogation sur les valeurs. Le but est de renforcer cette dimension. Concrètement, le projet concerné vise à l'organisation avec des étudiants volontaires d'un voyage à Auschwitz articulé ensuite à l'expression artistique des réflexions que cette expérience a suscitées auprès d'eux.

\section{Importance de ces logiques au sein des projets}

1. Le tableau qui suit objective la présence de ces logiques d'innovation au sein des projets.

\begin{tabular}{|c|c|c|c|c|}
\hline $\begin{array}{c}\text { Logique de } \\
\text { libération de } \\
\text { l'innovation }\end{array}$ & $\begin{array}{c}\text { Logique de } \\
\text { remédiation }\end{array}$ & $\begin{array}{c}\text { Logique des } \\
\text { compétences }\end{array}$ & $\begin{array}{c}\text { Logique de } \\
\text { l'intégration }\end{array}$ & $\begin{array}{c}\text { Logique de la } \\
\text { citoyenneté }\end{array}$ \\
\hline 2 & 5 & 10 & 6 & 1 \\
\hline
\end{tabular}

Il apparaît que la logique des compétences est la plus prégnante, suivie de la logique de l'intégration et de celle de la remédiation, les logiques de libération de l'innovation et de citoyenneté étant plus marginalement représentées. Ce qui pousse des enseignants universitaires à déposer un projet en réponse à un appel à expérience innovante tient donc en premier lieu à une volonté de voir la qualité des apprentissages s'améliorer via l'instauration d'une pédagogie active visant au développement de compétences. Il s'agit d'acquérir des moyens afin de proposer des dispositifs pédagogiques qui s'éloignent du cours magistral. La deuxième motivation des enseignants vise à rompre avec le cloisonnement des enseignements. Repenser collégialement un programme pour définir un projet de formation commun, faire des ponts entre les cours, accentuer l'interdisciplinarité sont au cœur de ce que les enseignants jugent qu'il est impératif de réaliser aujourd'hui pour lutter contre un cloisonnement délétère des apprentissages chez les étudiants. Enfin, ce n'est pas un hasard dans le contexte universitaire belge francophone où la première année se caractérise par des taux d'échecs très élevés, la troisième logique de motivation repose sur le principe de remédiation. La dimension innovante tient ici dans le recours aux TICe souvent dans une perspective d'auto-apprentissage en ligne. Autre constat, le tableau montre que les projets sont parfois habités par plusieurs logiques. En réalité, il s'agit essentiellement de projets qui articulent la logique des compétences et celle de l'intégration. Parmi les dix projets qui valorisent les méthodes actives, cinq adoptent également la logique de l'intégration. Généralement, il s'agit de méthodes fondées sur l'analyse de cas ou de problèmes dont la résolution implique la mobilisation conjointe de plusieurs disciplines. 


\section{Discussion}

Derrière la diversité des mutations qui traverse l'enseignement universitaire, cette contribution visait à rendre intelligibles quelques «logiques d'innovation pédagogique » au départ des motivations exprimées par les promoteurs de projets, des insuffisances qu'ils pointent et des formes des dispositifs proposés. Si une série de publications tentent d'identifier les tendances du changement pédagogique qui touchent aujourd'hui les universités, ou les caractéristiques des programmes innovants, peu, parmi elles, nous semble partir des représentations des acteurs quant aux limites de l'enseignement universitaire et aux solutions à mettre en place pour comprendre la nature de ces changements. Or, la volonté transversale d'«améliorer les apprentissages » que la littérature met généralement en avant derrière le développement de l'innovation pédagogique s'ancre en réalité dans des diagnostics variés quant aux difficultés perçues. Par ordre d'importance, les trois grandes limites de l'enseignement universitaire soulevées dans cette recherche par les promoteurs de projets sont: 1. la faible qualité et la superficialité des apprentissages, 2. le cloisonnement des apprentissages et 3. les échecs des étudiants en première année. Derrière ces difficultés apparaissent des familles d'innovations prônées : des méthodes actives qui visent les compétences et les savoirs mieux ancrés, des projets d'intégration d'enseignements, des outils de remédiation en ligne destinés à diminuer l'échec en première année que les cours ex cathedra ne savent résorber. Les TICe ne se résument toutefois pas à la remédiation. Il serait sans doute intéressant ici de saisir la façon dont les TICe se déclinent en regard des diverses logiques ; élément qui n'a pas été poussé plus avant dans cette étude.

\section{Conclusion}

Au départ d'une analyse de dossiers soumis en réponse à un appel institutionnel à expériences innovantes, cet article a permis d'identifier cinq logiques fondamentales qui sous-tendent les velléités de changement des enseignants et à évaluer leur présence dans le corpus analysé.

Développer une analyse compréhensive fondée sur les ressorts fondamentaux qui les poussent au changement (perception des insuffisances, des solutions, des dispositifs à mettre en place) tout en dégageant de façon idéal-typique les grandes logiques qui, de façon transversale aux disciplines, caractérisent les velléités des innovateurs permet de rendre intelligible ce qui travaille aujourd'hui les acteurs de l'université sur un plan de l'enseignement. Une telle analyse nous semble être de nature à contribuer aux réflexions théoriques sur ce que l'on appelle 
l'innovation pédagogique en apportant de la clarté au sein d'une dynamique complexe, plurielle, faite d'un foisonnement souvent hétérogène d'initiatives que l'on ne saurait résumer ni à sa dimension technologique, ni à la simple quête d'une amélioration des apprentissages. Elle peut également contribuer à pallier certains constats récemment mis en avant par Viaud (2015) selon lesquels l'enseignement reste une préoccupation secondaire des enseignants universitaires et que ceux-ci méconnaissent les évolutions qui travaillent l'enseignement universitaire ainsi que les initiatives portées par leurs collègues. Proposer, à l'échelle d'une institution, une analyse des tendances de l'innovation en partant des réflexions des enseignants quant à ce qui dysfonctionne et ce qu'il faudrait mettre en place constitue un potentiel effet de miroir qui permet d'alimenter la réflexion sur l'enseignement et de soutenir les velléités des innovateurs.

\section{Références bibliographiques}

Béchard, J.-P. et Pelletier, P. (2001). Développement des innovations pédagogiques en milieu universitaire : un cas d'apprentissage organisationnel. Dans D. Raymond, Nouveaux espaces de développement professionnel et organisationnel (p. 131-149). Université de Sherbrooke : Éditions du CRP.

Bédard, D. et Béchard, J.-P. (2009). L'innovation pédagogique dans le supérieur : un vaste chantier. Dans D. Bédard et J.-P. Béchard (dir.), Innover dans l'enseignement supérieur (p. 29-43). Paris : Presses universitaires de France.

Cros, F. (2001). L'innovation scolaire. Paris : Institut National de Recherche Pédagogique.

Cros, F. (2004). L'innovation scolaire aux risques de son évaluation. Paris : L'Harmattan.

Prégent, R., Bernard, H. et Kozanitis, A. (2009). Enseigner à l'université dans une approche programme. Montréal : Presses internationales polytechniques.

Rege-Colet, N. (2007). Évolution, révolution ou renouvellement de la pratique d'enseignement. Mais qu'est-ce qui change vraiment? Conférence donnée lors de la Journée d'échanges et de réflexion sur l'évolution des pratiques enseignantes à l'université Vendredi 30 novembre 2007. Académie Universitaire Wallonie - Bruxelles

Rege-Colet N. et Romainville, M. (2006). La pratique enseignante en mutation à l'université. Bruxelles : De Boeck.

Roegiers, X. (2012). Quelles réformes pédagogiques pour l'enseignement supérieur ? Placer l'efficacité au service de l'humanisme. Bruxelles : De Boeck.

Romainville, M. (2008). Et si on arrêtait de tirer sur les compétences. Indirect, 10, 31-43.

Lison, C., Bédard, D., Beaucher, C. et Trudelle, D. (2014). De l'innovation à un modèle de dynamique innovationnelle en enseignement supérieur. Revue internationale de pédagogie de l'enseignement supérieur, 30(1). https://doi.org/10.4000/ripes.771

Paillé, P. et Muchielli, A. (2008). L'analyse qualitative en sciences humaines et sociales. Paris : Armand Colin. 
Schnapper, D. (1999). La compréhension sociologique. Démarche de l'analyse typologique. Paris : PUF.

Tardif, J. (2006). L'évaluation des compétences. Documenter le parcours de développement. Montréal : Chenelière Éducation.

Viaud, M.-L. (2015). Les innovateurs silencieux. Histoire des pratiques d'enseignement à l'université depuis 1950. Grenoble : Presses universitaires de Grenoble. 